\title{
The Difficulty of Defining Sensitive Data-The Concept of Sensitive Data in the EU Data Protection Framework - CORRIGENDUM
}

\author{
Paul Quinn and Gianclaudio Malgieri
}

https://doi.org/10.1017/glj.2021.79, Published by Cambridge University Press, 19 January 2022

Keywords: Sensitive Data; Data Protection; GDPR; Privacy; Health Data

Gianclaudio Malgieri - one of the authors of this paper - was inadvertently omitted in the author line of the article as originally published.

The article has now been updated to include the full authorship details, which are provided below:

Paul Quinn ${ }^{1}$ and Gianclaudio Malgieri ${ }^{2}$

1. Law, Science, Technology \& Society, Vrije Universiteit Brussel, Brussels, Belgium

2. EDHEC Augmented Law Institute, Lille, France

Both individuals have confirmed that they meet ICMJE criteria for authorship.

\section{Reference}

Quinn, P., and Malgieri, G. (2021). The Difficulty of Defining Sensitive Data-The Concept of Sensitive Data in the EU Data

Protection Framework. German Law Journal, 22(8), 1583-1612. doi: 10.1017/glj.2021.79

Cite this article: Quinn P, Malgieri G (2022). The Difficulty of Defining Sensitive Data-The Concept of Sensitive Data in the EU Data Protection Framework - CORRIGENDUM. German Law Journal 23, 688. https://doi.org/10.1017/glj.2022.11 